\title{
Comparative Cytogenetics of Some Species of Crocidura (Insectivora) with $2 n=40$
}

\author{
Larisa S. Biltueva ${ }^{1}$, Polina L. Perelman ${ }^{1}$, Margarita B. Rogatcheva ${ }^{1,3 *}$, \\ Elena L. Fedorova ${ }^{1}$, Nadezhda V. Vorobieva ${ }^{1}$, Masashi Harada ${ }^{2}$, \\ Kazuyuki Mekada ${ }^{3}$, Sen-Ichi Oda ${ }^{3}$ and \\ Alexander S. Graphodatsky ${ }^{1}$ \\ ${ }^{1}$ Institute of Cytology and Genetics, Russian Academy of Sciences, Novosibirsk, 630090, Russia \\ ${ }^{2}$ Laboratory of Experimental Animals, Medical School, Osaka City University, Osaka 545-8586, Japan \\ ${ }^{3}$ Laboratory of Animal Management, School of Agricultural Sciences, Nagoya University, Nagoya 464-8601, Japan
}

Accepted June 9, 1999

\begin{abstract}
Summary A GTG-banding was used for a detailed comparative analysis of two 40-chromosome Crocidura species, Crocidura gueldenstaedtii and Crocidura dsinezumi. A high level of chromosomal homology was detected. A complex 520-bp EcoRI tandem repeat was isolated from C. dsinezu$m i$. Using fluorescence in situ hybridization (FISH), this repeat was localized to centromeric regions of chromosomes in both Crocidura species.
\end{abstract}

Key words Crocidura dsinezumi, Crocidura gueldenstaedtii, CDZ repeat, FISH, Comparative analysis, Chromosomal homology.

Today, there are more than 150 known species of the genus Crocidura. Among the 40 that have been karyotyped, only a few have been analyzed using C- and G-banding techniques. In the genus Crocidura the chromosome numbers have been found to range from 22 (Crocidura pergrisea) in Palearctic and Oriental species to 60 (Crocidura bicolor) in African species (Maddalena and Ruedi 1994). Most of the African species have a high diploid number (more than 40), whereas all Palearctic and Oriental species contain 40 chromosomes or less. Chromosomal comparison using differential staining of several Crocidura species assumed an ancestral karyotype of the genus having 36-40 chromosomes (Maddalena and Ruedi 1994). The evolution of the genus Crocidura may have proceeded in two opposite directions: the low chromosome Eurasian species may have evolved with a reduction in diploid number (Robertsonian and tandem fusion), while in the higher chromosomal African species, evolution may have occurred from an increase in both diploid and fundamental numbers due to fission (Graphodatsky et al. 1988, Maddalena and Ruedi 1994, Harada et al. 1985).

Many of the Eurasian species of Crocidura have 40 chromosomes. Graphodatsky et al. (1988) regarded such a number as primitive among the Palearctic-Oriental (Eurasian) species. To better understand the ancestral karyotype, a comparison of 40-chromosome species would be useful. For three Palearctic 40-chromosome species, Crocidura suaveolens, Crodidura sibirica and Crocidura gueldenstaedtii, G- and C-banding revealed complete homology of the autosome sets and the X chromosomes (Graphodatsky et al. 1988). Differing only slightly phenotypically while appearing to have the same karyotype, these three species are often classified as a single group named Suaveolens (Graphodatsky et al. 1988, Zaitsev 1989). The G- and C-band karyotypes of the Oriental 40chromosome Crocidura dsinezumi has been previously described by Harada et al. (1985).

Here we present a comparison of GTG- and C-band karyotypes of the Oriental C. dsinezumi and Palearctic C. gueldenstaedtii (the Suaveolens group). A 520-bp EcoRI complex tandem repeat

* Corresponding author: Margarita B. Rogatcheva, Laboratory of Animal Management, School of Agricultural Sciences, Nagoya University, Nagoya 464-8601, Japan. E-mail:rogatchrita@hotmail.com 
was isolated from C. dsinezumı. Using fluorescence in situ hybridization (FISH) it was localized to the chromosomes of both Crocidura species.

\author{
Material and methods
}

\title{
Banding analysis
}

Metaphase chromosomes were prepared from bone marrow cells that were suspended in 199 tissue culture medium supplemented with $10 \%$ calf serum and incubated for $2 \mathrm{~h}$ at $37^{\circ} \mathrm{C}$ with colcemid $(0.02 \mu \mathrm{g} / \mathrm{ml})$ for $30 \mathrm{~min}$. The cells were swollen in $0.04 \mathrm{M} \mathrm{KCl}$ for $15 \mathrm{~min}$, fixed in ice-cold methanol-acetic acid fixative, spread on moist cold slides and air-dried. G-band staining was performed according to the method of Seabright (1971), as modified by Graphodatsky and Radjabli (1988). Slides were treated with a $0.025 \%$ trypsin solution in isotonic saline for $1-2 \mathrm{~min}$ at room temperature, rinsed in $2 \times \mathrm{SSC}$ and stained for $5 \mathrm{~min}$ in $4 \%$ Giemsa solution. C-band staining followed the modified version of Sumner (1972). Slides were incubated in $5 \% \mathrm{Ba}(\mathrm{OH})_{2}$ for $5 \mathrm{~min}$, then in $2 \times \mathrm{SSC}$ for $1 \mathrm{~h}$ at $62.5^{\circ} \mathrm{C}$ before staining in $4 \%$ Giemsa for $30 \mathrm{~min}$.

\section{DNA isolation and sequencing}

DNA was isolated from liver and a restriction enzyme digestion performed using standard procedures (Sambrook et al. 1989). Highly repeated sequences were 1solated by digestion of the total DNA of $C$. dsinezumi with the restriction enzyme EcoRI resulting in a 520-bp fragment. The DNA fragment was isolated by electroelution, cloned in the EcoRI site of a pUC19 vector and transformed into an E. coli strain DHSa. Transformants were identified and manually sequenced using appropriate primers by the dideoxynucleotide chain-terminal method (Sanger et al. 1977) and the Sequenase Version 2.0 system (USB).

The DNA fragment was signed as CDZ and its genomic sequence was deposited in the EMBL Database under Accession Number Y14806.

\section{Southern blot hybridization}

C. dsinezumi was tested for repeat sequences by Southern blot analysis. $5 \mu \mathrm{g}$ of DNA was digested with EcoRI, fractionated on $1.5 \%$ agarose gel, and transferred to a Hybond $\mathrm{N}$ membrane (Amersham). Plasmid probes were labeled with ${ }^{32} \mathrm{P}$ by random priming. Hybridizations were performed at $55^{\circ} \mathrm{C}$ in $6 \times \mathrm{SSC}, 0.5 \% \mathrm{SDS}, 100 \mathrm{mg} / \mathrm{ml}$ heparin and $100 \mathrm{mg} / \mathrm{ml}$ salmon sperm DNA, and washed at $65^{\circ} \mathrm{C}$ in $0.1 \times \mathrm{SSC}$ and $0.1 \%$ SDS.

Repeat DNA bands corresponding to the cloned fragment and its multimers were taken as DNA ladders.

\section{FISH}

Fluorescence in situ hybridızation (FISH) was performed accordıng to Pinkel et al (1986). Hybridization of a biotın-labeled probe of CDZ repeat to Crocidura chromosomes was detected with fluorescein-conjugated avidin. Chromosomes were counterstained with propidium iodide. Photographs were taken on Kodak 400 ASA film.

\section{Results and discussion}

\section{Comparative analysis of $G$ - and C-banded chromosomes}

G- and C-banded chromosomes of $C$. dsinezumi (Figs. 1b, 2b) were arranged according to Harada et al. (1985), and the chromosomes of C. gueldenstaedtii (Figs. 1a, 2a) according to Graphodatsky et al. (1988). For comparative analysis (Fig. 3), we used GTG-banded chromosomes of C. dsinezumi and C. gueldenstaedtii at the same level of resolution. 



Fig. 1. G-banded karyotypes of C. gueldenstaedtii (a) and C. dsinezumi (b).

The centromeric C-heterochromatin blocks of different sizes were located on most of the autosomes and on the $\mathrm{X}$ chromosome of $C$. gueldenstaedtii (Fig. 2a). All chromosomes of $C$. dsinezumi (Fig. 2b), except the submetacentrics 4, 5 and 7, displayed small blocks of centromeric C-heterochromatin. The $\mathrm{X}$ chromosome of $C$. dsinezumi had an additional telomeric $\mathrm{C}$-band relative to the C. gueldenstaedtii X chromosome. The Y chromosomes of both species were uniformly dark.

Comparison of G-band karyotypes revealed a high level of homology between the chromosomes of $C$. dsinezumi and C. gueldenstaedtii (Fig. 3). Nine of the 19 pairs of autosomes of $C$. dsinezumi were identical to nine pairs of chromosomes of $C$. gueldenstaedtii. Submetacentrics 5, 2 and 3 of $C$. dsinezumi were homologous to submetacentrics 1, 3 and 4 of $C$. gueldenstaedtii, respectively. Acrocentrics $9,11,10,13,15$, and 17 of $C$. dsinezumi were identical to acrocentrics 6,10 , $12,16,14$, and 17 of $C$. gueldenstaedtii, respectively. The q-arm of subtelocentric 2 of $C$. gueldenstaedtii was homologous to $C$. dsinezumi acrocentric 12 , whereas the p-arm of subtelocentric 2 was 


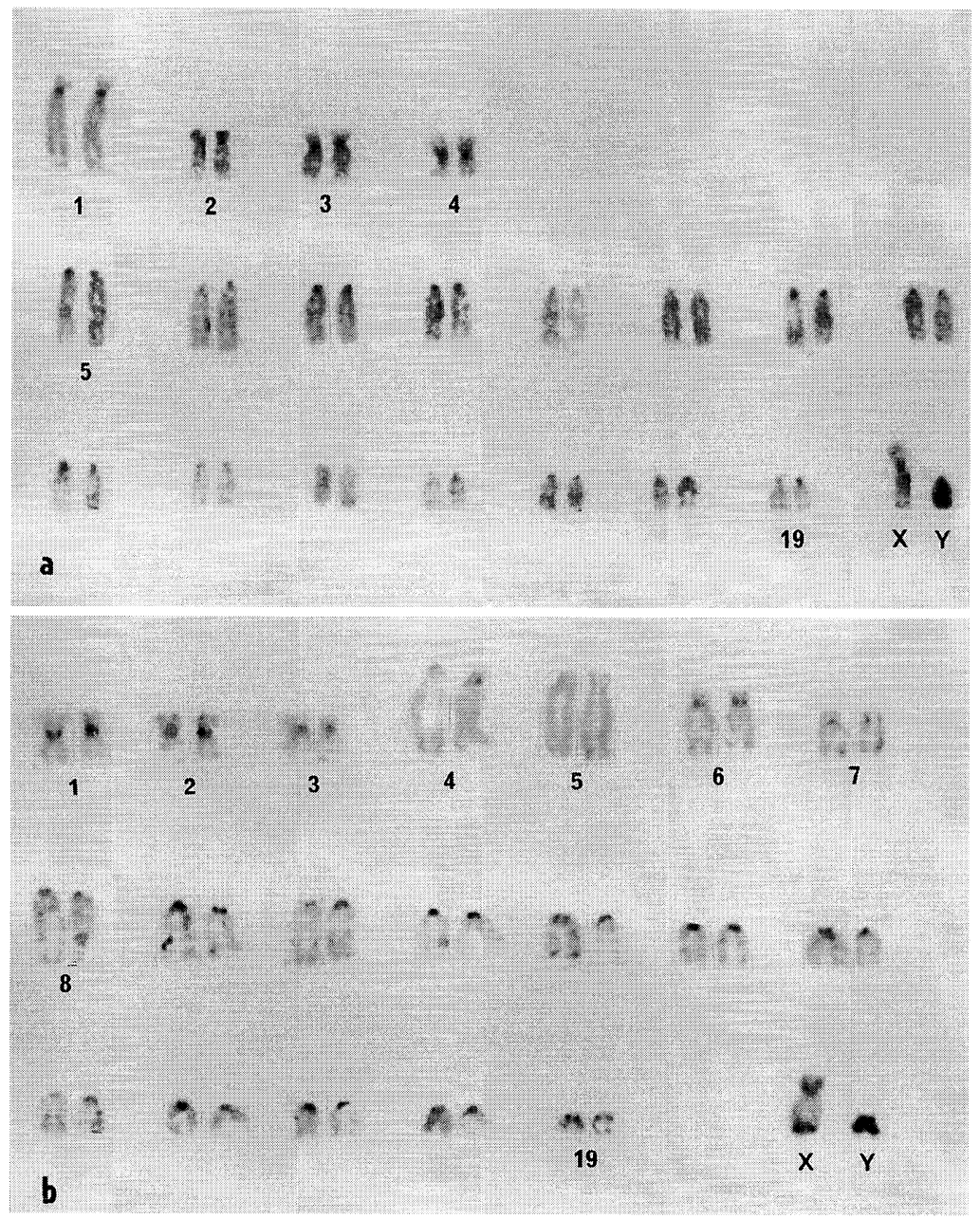

Fig. 2. C-banded karyotypes of C. gueldenstaedtii (a) and C. dsinezumi (b). Since it is impossible to distinguish the $\mathrm{C}$-banded acrocentrics, only the bigger and smallest acrocentrics were numbered.

completely heterochromatic.

The following arm homology was detected. The q-arms of the C. dsinezumi chromosomes 4 and 6 were homologous to acrocentrics 5 and 9 of $C$. gueldenstaedtii, respectively. The $C$. dsinezu$m i$ chromosomes 16 and 19 appeared to be similar to chromosome 11 of $C$. gueldenstaedtii.

We found monobrachial homology between the $C$. dsinezumi chromosomes 1 and 18 and chromosomes 7 and 15 of $C$. gueldenstaedtii. The q-arm of chromosome 1 of $C$. dsinezumi was homologous to acrocentric 15 of $C$. gueldenstaedtii, whereas the p-arm of chromosome 1 and acrocentric 18 of $C$. dsinezumi were similar to chromosome 7 of $C$. gueldenstaedtii.

The remaining three pairs of chromosomes displayed extensive regions of homology. $C$. dsinezumi chromosome 7 had a similar banding pattern to the major part of acrocentric 13 of $C$. gueldenstaedtii. The banding pattern of the proximal part of the chromosome 8 of $C$. dsinezumi corresponded to that of the distal part of chromosome 18 of $C$. gueldenstaedtii, whereas the rest of $C$. dsinezumi chromosome 8 was homologous to $C$. dueldenstaedtii chromosome 8 . The distal two thirds of acrocentric 14 of $C$. dsinezumi corresponded to acrocentric 19 of $C$. gueldenstaedtii except for an inverted position.

The small proximal parts of $C$. dsinezumi chromosomes 4,6 and 14 were probably homolo- 


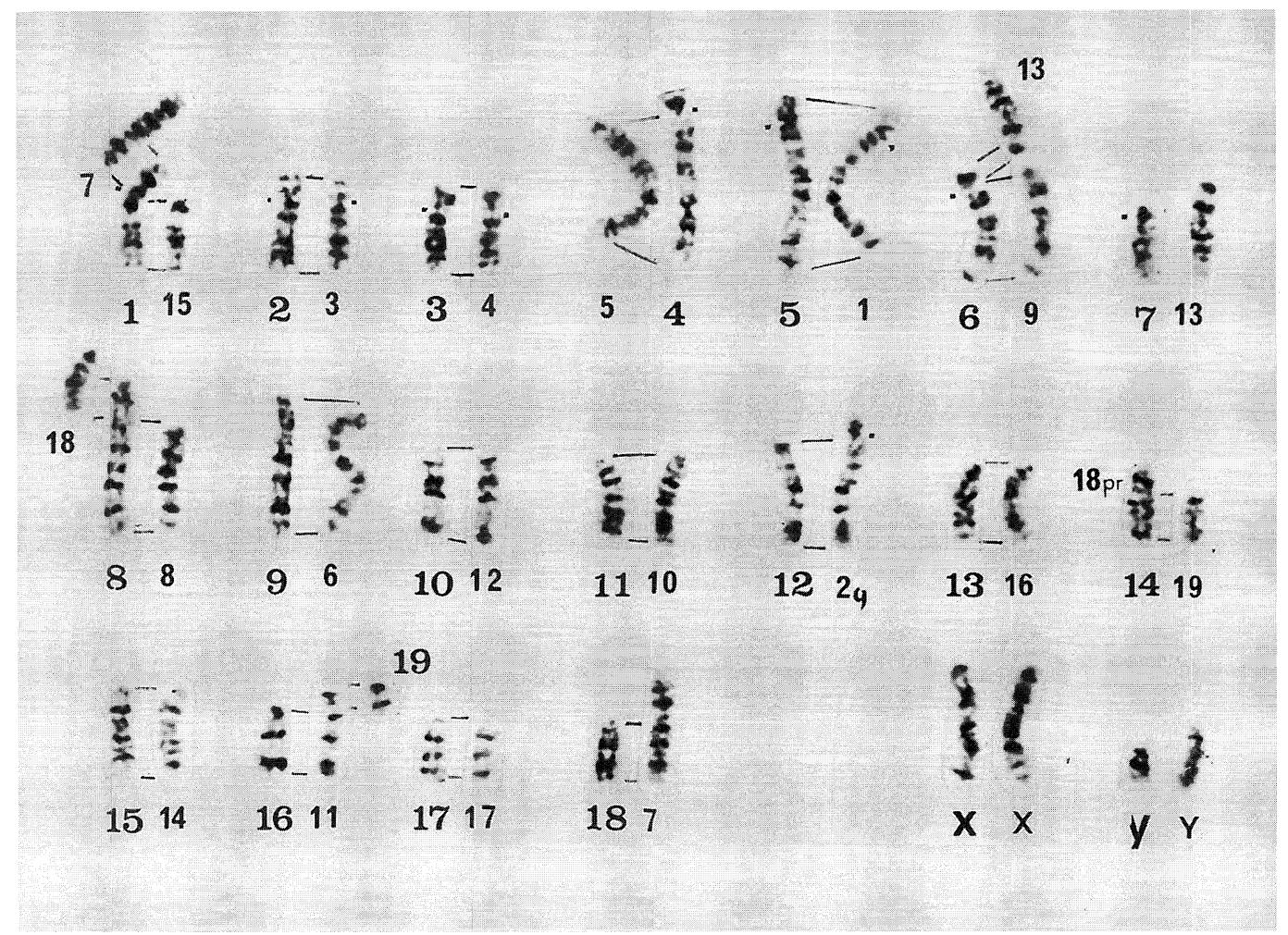

Fig. 3. Comparison of haploid complements of C. dsinezumi (large fiigures) andC. gueldenstaedtii (small fiigures). Outside bars indicate regions of homology

gous to the proximal parts of $C$. gueldenstaedtii chromosomes 13 and 18 , but they were too small for this to be determined in details.

The GTG-banding pattern of the X chromosomes differed substantially. Several rearrangements such as perecentric inversion and centromeric shift have possibly taken place in the evolution of the X chromosome. The Y chromosomes were both acrocentrics, with the Y chromosome of $C$. gueldenstaedtii being twice as long as the $\mathrm{Y}$ chromosome of $C$. dsinezumi. Such a size difference could be due to the variation in the amount of C-heterochromatin (Harada et al. 1985, Graphodatsky et al. 1988, this study).

Thus, the GTG-banding revealed a high level of homology between Oriental and Palearctic 40chromosome species. The regions of homology and homologous chromosomes that have remained conserved during chromosomal evolution could be elements of Eurasian Crocidura ancestral karyotype. If the ancestral karyotype consisted of more than 40 chromosomes, the chromosome evolution may have involved only tandem fusion and whole arm reciprocal translocations. If the ancestral karyotype consisted of 40 chromosomes, more rearrangements would have been needed, including not only fusion and whole arm reciprocal translocations, but also fission. To clarify this, a greater number of species must be compared.

Most of the conserved chromosomes, or part of the chromosomes detected, are present also in the hypothetical ancestral karyotype proposed by Maddalena and Ruedi for the whole genus Crocidura (1994). 


$\begin{array}{llllll}\text { GAATTCACCT } & \text { GTGGTAAAAA } & \text { GATTCTTCTC } & \text { CTTTCTTGAA } & \text { ACtGCTTT GT } & \text { CGAAGCTTGT } \\ \text { ACAACTCGGC } & \text { AGTGATCTCT } & \text { GTGTTCTTCC } & \text { TACAGAGTTC } & \text { TGAGCACATT } & \text { TGCGTGGCCG } \\ \text { ACGAACATTA } & \text { CACAATTGAG } & \text { AACTTAACAT } & \text { GCGGTTTCA } & \text { TCAAGATTCT } & \text { TCAATTTTCA } \\ \text { GTTTCTCATT } & \text { TCACTGTTCT } & \text { TTTCCCTTCT } & \text { GTTCTAATGC } & \text { AATGTGTATG } & \text { TTTCTATATA } \\ \text { TTCTGCGCAT } & \text { TCAGTCATCT } & \text { CCTTCTTTGA } & \text { TTTTGATGTG } & \text { TTACTGATGT } & \text { TCTTTCTTCT } \\ \text { TTGCTCATAA } & \text { CTTTACACCC } & \text { CGAAGTGTTC } & \text { TCTGTGTTAT } & \text { TCTAATGCTG } & \text { TCTTAATACT } \\ \text { TTTGCATCTC } & \text { CCTTTAAAAA } & \text { CTACACATT } & \text { GACAGCAGAA } & \text { AATGTTGTGT } & \text { TCTTCATAAT } \\ \text { ACTTGATTAT } & \text { TCACGTTTGA } & \text { TTTCATTGAA } & \text { TGCAAGTTC } & \text { TGTTGTAATT } & \text { TGATGTGAAT } \\ \text { ATTGCTTGTT } & \text { TTTACTGTAT } & \text { TCGTTATCT } & \text { CGGTTT } & & \end{array}$

Fig. 4. The primary structure of the $\mathrm{CDZ}$ repeat, isolated from C. dsinezumi.
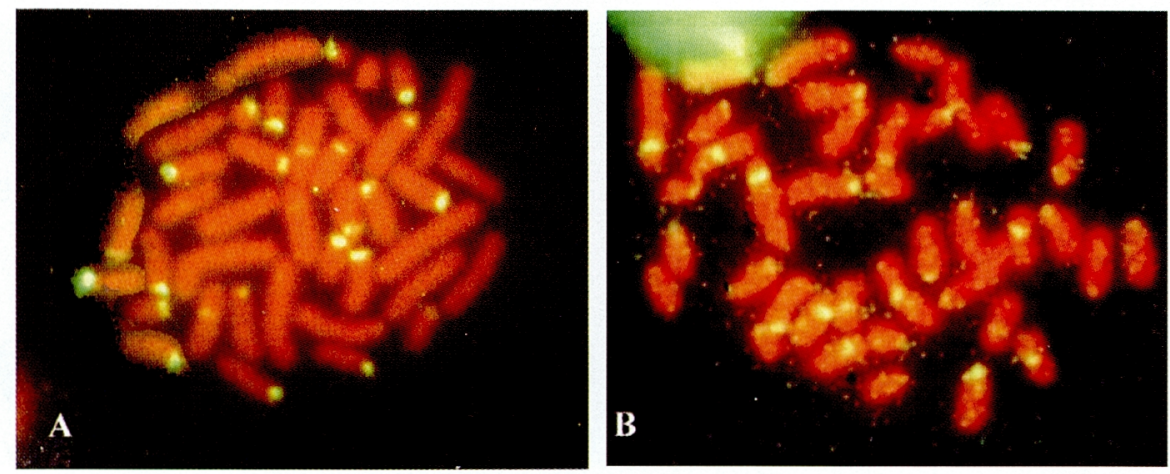

Fig. 5. Metaphase chromosomes following flluorescencein situ hybridization with the CDZ repeat DNA probe: C. dsinezumi (A), C. gueldenstaedtii (B).

array in C. dsinezumi and C. gueldenstaedtii. It was absent in the genomes of Sorex araneus and Suncus murinus (data not shown). The repeated nucleotide sequence is shown in Fig. 4. No homology was found between CDZ crocidura-specific repeats and any known repeated sequences isolated from Insectivores or any other mammals. The CDZ repeat appears to be genus specific, or even subgenus specific, regarding Suncus as subgenus of genus Crocidura.

Results of the in situ hybridization of the CDZ repeat to Crocidura Chromosomes (Fig. 5) demonstrate that $C$. dsinezumi and $C$. gueldenstaedtii share the same centromeric repeat. Most of the chromosomes of $C$. dsinezumi (Fig. 5A) displayed clear signals in their centromeric regions. In C. gueldenstaedtii the $\mathrm{CDZ}$ repeat was also located on the centromeric regions of chromosomes. The $\mathrm{X}$ and $\mathrm{Y}$ chromosomes of both species had signals only in the centromeric regions.

Both cytogenetic and molecular data favor C. gueldenstaedtii and C. dsinezumi having monophyletic origin and a recent common ancestry.

\section{Acknowledgments}

We are grateful to Dr. David Walsh for reading this manuscript and offering helpful comments. This work was supported in part by a research grant from the Russian Fund for Basic Research and Biodiversity Program (L.S.B., P.L.P., E.L.F., N.V.V. and A.S.G.) and from the Japanese Society for Promotion of Sciences (M.B.R.).

References

Graphodatsky, A. S. and Radjabli, S. I. 1988. Chromosomes of farm and laboratory animals. Novosibirsk, Nauka (in Russian). 
-, - Sharshov, A. V. and Zaitsev, M. V. 1988. Karyotypes of five Crocidura species of the USSR fauna. Tsitologya 30: 1247-1255 (in Russian with English summary).

Harada, M., Yosida, T. H., Hattori, S. and Takada, S. 1985. Cytogenetical studies on Insectivora. Karyotype comparison of two Crocidura species in Japan. Proc. Japan. Acad. 61, ser. B: 371-374.

Maddalena, T. and Ruedi, M. 1994. Chromosomal Evolution in the Genus Crocidura (Insectivora: Soricidae) In: Merritt, J. F., Kirkland, G. L. and Rose, R. K. (eds.) Advances in Biology of Shrews. Pittsburgh. Carnegie Mus. Natur. Hist.: 335-344.

Pinkel, D., Straume, T. and Gray, J. W. 1986. Cytogenetic analysis using quantitative, high-sensitivity fluorescence hybridization. Proc. Natl. Acad. Sci., USA, 83: 2934-2938.

Sambrook, J., Fritsch, E. F. and Maniatis, T. 1989. Molecular Cloning a Laboratory Manual (2nd ed.). Cold Spring Harbor Laboratory Press, Cold spring Harbor, New York.

Sanger, F., Nicklen, S. and Coulson, A. R. 1977. DNA sequencing with chain-terminating inhibitors. Proc. Natl. Acad. Sci. USA 74: 5463-5467.

Seabright, M. 1971. A rapid banding technique for human chromosomes. Lancet No 7731: 971-972.

Sumner, A. 1972. A simple method for demonstrating centromeric heterochromatin. Exp. Cell Res. 75: 304-306.

Zaitsev, M. V. 1989. Species composition and questions of systematic of white-toothed shrews (Mammalia, Insectivora) of the Fauna of USSR. Proc. Zool. Inst. 243: 3-46. 